Reprod. Nutr. Dévelop., 1983, 23 (3), 607-623.

\title{
Influence des ciliés du rumen sur l'utilisation digestive de différents régimes riches en glucides solubles et sur les produits terminaux formés dans le rumen. II. - Régimes contenant de l'inuline, du saccharose et du lactose (1)
}

\author{
J.-P. JOUANY, J. SENAUD $\left(^{*}\right)$
}

Laboratoire de la Digestion des Ruminants, I.N.R.A., Theix, 63110 Beaumont, France.

${ }^{*}$ ) L.A. CNRS $n^{\circ} 138$, Biologie des protistes, Université de Clermont II, 63170 Aubière, France.

Summary. Effect of rumen ciliates on the digestive utilization of various carbohydraterich diets and on the end-products formed in the rumen. II. Utilization of inulin, saccharose and lactose.

Three diets rich in inulin, saccharose and lactose, respectively, were given to 10 rumen-fistulated sheep. Two animals were defaunated, two were inoculated with either Polyplastron multivesiculatum or Entodinium sp., and two others were inoculated with both. The latter two were bred in conventional conditions.

All animals ingested the same amounts of carbohydrates in the three diets $121-22 \mathrm{~g} / \mathrm{kg}$ $\mathrm{P}^{0.75} /$ day). Dietary nitrogen content was similar (table 1). The ciliate population was improved with the inulin diet (fig. 1 ; table 2). With a mixed population, the Entodinium $s p$. genus was always predominant. Holotrich protozoa (mainly /sotricha) in the rumen of the conventional sheep represented 15 to $30 \%$ of the total ciliate biomass, indicating that they were able to metabolize these soluble sugars. We also observed that $P$. multivesiculatum can ferment cellulose and all the soluble carbohydrates proposed in these diets. However, Entodinium sp. development occurred mainly in the presence of the sugars produced during carbohydrate hydrolysis by other ciliates or bacteria.

The highest organic matter digestibility, noted in faunated animals (table 3 ) was confirmed by the VFA concentration in the rumen (table 4). This could be explained either by an activation of bacterial metabolism due to predation or by the direct effect of ciliates on fermentations, or both.

Modifications in the VFA composition varied with ciliate inoculation, showing that ciliate metabolism may vary with the nature of the energy in the diet or that the observed results depended on various opposite effects in which the intensity of each component was influenced by the diet. In general, the acetic acid molar proportion increased and propionic acid decreased when there was a considerable Entodinium sp. population. The effect on butyric acid was low with these diets. Higher ammonia and lactic acid concentrations were observed in the rumen of faunated than defaunated sheep, irrespective of the ciliate inoculum.

(1) Utilisation des glucides pariétaux (cellulose et hemicelluloses) et de l'amidon. Reprod. Nutr. Dévelop., 1982, 22, 735-752. 


\section{Introduction.}

La ration de base des Ruminants domestiques est constituée de fourrages qui contiennent une proportion variable de glucides pariétaux dont la cellulose est, quantitativement, le représentant le plus important (sa teneur varie de $15 \%$ de la matière sèche pour l'herbe jeune, à plus de $40 \%$ pour la paille). Pour les animaux en production, on distribue, en complément, des aliments riches en énergie. Ces derniers ont une teneur élevée en glucides facilement fermentescibles pouvant provenir de céréales (cas de l'amidon) ou d'aliments tels que la betterave (riche en saccharose), le topinambour (riche en inuline) ou encore le lactosérum (riche en lactose) dont I'utilisation dans la ration des Ruminants est récente (Thivend, 1978). La digestion de l'ensemble de ces glucides a lieu surtout dans le rumen, sous l'action conjuguée des bactéries et des protozoaires. Grâce à l'obtention d'animaux à faune contrôlée, nous avons pu préciser, au cours d'un premier travail (Jouany et Senaud, 1982) le rôle respectif des protozoaires ciliés du rumen appartenant aux genres Entodinium et Polyplastron dans la digestion de régimes riches en cellulose ou en amidon.

L'objectif de ce travail a consisté à mesurer le développement des différentes populations de protozoaires puis à déterminer les caractéristiques fermentaires de la digestion de ces différents glucides dans le rumen, ainsi que la digestibilité des principaux constituants des rations étudiées, chez des animaux qui étaient soit défaunés, soit mono-inoculés avec l'espèce Polyplastron multivesiculatum ou avec le genre Entodinium, soit bi-inoculés avec les deux ciliés, soit conventionnels.

\section{Matériel et méthodes.}

Aliments. - Les trois régimes décrits dans le tableau 1 ont été étudiés dans l'ordre suivant :

1) Le régime "inuline » qui était constitué principalement de topinambours, distribués sous forme de cossettes à raison de $623 \mathrm{~g}$ de $\mathrm{MS}$ par jour et par animal, de foin de prairie naturelle haché en brins d'environ $10 \mathrm{~cm}(281 \mathrm{~g}$ de MS par jour et par animal) et de tourteau d'arachide (91 g de MS par jour et par animal).

2) Le régime " saccharose " qui comprenait des betteraves hachées en cossettes (625 g de MS par jour et par animal), du foin de prairie naturelle identique à celui du précédent régime (134 g par jour et par animal), et du tourteau d'arachide $192 \mathrm{~g}$ de MS par jour et par animal).

3) Le régime "lactose " qui était composé d'ultrafiltrat de lactosérum $1417 \mathrm{~g}$ de MS par jour et par animal), du même foin de prairie naturelle que celui précédemment utilisé, à raison de $457 \mathrm{~g}$ par jour et par animal, et de $122 \mathrm{~g}$ de MS de tourteau d'arachide, distribués chaque jour à chaque animal.

Les régimes étaient isoazotés (tabl. 1). Les quantités de glucides ingérées ont peu varié entre régimes : 20,$9 ; 21,0$ et $22,1 \mathrm{~g}$ par $\mathrm{kg} \mathrm{P}^{0,75}$ respectivement d'inuline, de saccharose et de lactose. Les rations étaient distribuées aux animaux en repas égaux, 2 fois par jour, à 8 h 30 et 16 h 30 . 
TABLEAU 1

Composition des régimes

\begin{tabular}{|c|c|c|c|}
\hline Régimes & inuline & saccharose & lactose \\
\hline \multicolumn{4}{|l|}{ Composition centésimale } \\
\hline Foin de prairie naturelle (haché) & 28,2 & 15,8 & 45,9 \\
\hline Topinambours (en cossettes) & 62,1 & - & - \\
\hline Betteraves (en cossettes) & - & 73,4 & - \\
\hline Ultrafiltrat de lactosérum (en poudre) & - & - & 41,9 \\
\hline Tourteau d'arachide & 9,2 & 10,8 & 12,2 \\
\hline Total & 100,0 & 100,0 & 100,0 \\
\hline \multicolumn{4}{|l|}{ Composition chimique (\% MS) } \\
\hline Matière organique & 89,6 & 88,7 & 90,9 \\
\hline Cellulose* & 11,6 & 11,2 & 15,0 \\
\hline Inuline ${ }^{* *}$ & 27,4 & - & - \\
\hline Saccharose ${ }^{* *}$ & 6,5 & 28,6 & - \\
\hline Lactose ${ }^{* *}$ & - & - & 34,5 \\
\hline Matières azotées $(\mathrm{N} \times 6,25)$ & 14,1 & 14,9 & 14,8 \\
\hline PDIN*** & - & 9,7 & - \\
\hline $\mathrm{PDIE}^{* * *}$ & - & 8,4 & - \\
\hline
\end{tabular}

* Selon Jarrige (1961).

* Selon Jouany (1972) : l'inuline a été déterminée par dosage du fructose libéré après hydrolyse par $\mathrm{H}_{2} \mathrm{SO}_{4} 0,5 \mathrm{~N}$ à $60^{\circ} \mathrm{C}$ pendant 1 heure.

*** Les valeurs PDIE et PDIN de l'ultrafiltrat de lactosérum ne sont pas connues avec précision actuellement ; celles du topinambour n'ont pas été déterminées.

Schéma expérimental. - La conduite de l'expérience a été effectuée selon un schéma identique à celui décrit dans notre premier travail (Jouany et Senaud, 1982) dans lequel ont également été décrites les méthodes de mesures et d'analyses utilisées.

Traitement statistique des données. - Nous avons appliqué la méthode d'analyse de variance à une voie pour le traitement de lots de taille différente (Snedecor et Cochran, 1971), à l'étude statistique de l'effet des protozoaires (comparaison horizontale sur les tableaux 2-3-5-6) et des régimes (comparaison verticale). Le seuil de $5 \%(P<0,05)$ a été retenu pour la mise en évidence de différences significatives. En outre, nous avons effectué une analyse multidimentionnelle sur le plan factoriel suivant : 2 (effet Polyplastron) $\times 2$ (effet Entodinium) $\times 3$ (effet " régime $»)$ qui est décrite dans le tableau 4 .

Animaux. - Nous avons utilisé les mêmes animaux que ceux décrits dans notre première étude (Jouany et Senaud, 1982) : deux étaient défaunés $(-C$ ), deux autres étaient mono-inoculés avec la seule espèce Polyplastron multivesiculatum $(P)$, deux hébergaient le seul genre Entodinium $(E)$; deux étaient biinoculés avec les deux genres $(P+E)$, tandis que les rumens des deux derniers contenaient une faune mixte de type conventionnel $(+C)$. 


\section{Résultats.}

Description de la faune du rumen (tabl. 2, fig. 1 ).

Dans le rumen des animaux mono-inoculés c'est avec le régime « inuline » que les effectifs de $P$ et de $E$ ont été les plus importants $\left(14 \times 10^{3}\right.$ et $490 \times$ $\left.10^{3} / \mathrm{ml}\right)$. Ils ont été plus faibles avec le régime "lactose " $\left(6 \times 10^{3}\right.$ et $269 \times$ $\left.10^{3} / \mathrm{ml}\right)$ et surtout avec le régime " saccharose » $\left(4 \times 10^{3}\right.$ et $\left.183 \times 10^{3} / \mathrm{ml}\right)$. Les deux genres se sont généralement mieux développés lorsqu'ils étaient présents simultanément ; l'augmentation de $E$ a été particulièrement importante, celle de $P$ n'a été significativement améliorée que chez les animaux recevant le régime " saccharose ». La présence d'autres genres (Dasytricha et /sotricha) dans le rumen des animaux conventionnels a peu modifié la population de $E$ sauf dans le cas du régime " lactose » pour lequel ce genre a fortement augmenté au détriment de $P$.

Pour l'ensemble des régimes étudiés, la biomasse totale des ciliés représentée par leur volume a toujours été plus importante chez les animaux conventionnels qui ont reçu le régime " inuline " $(47 \mathrm{ml} /$ litre de contenu) par rapport à celle des animaux recevant du saccharose ou du lactose pour lesquels cette biomasse a été voisine $(27,5$ et $35,5 \mathrm{ml} /$ litre de contenu). La proportion de la biomasse représentée par les ciliés appartenant au genre Entodinium a toujours été dominante (de 60 à $80 \%$ ). Les ciliés appartenant à la famille des /sotrichidae ont constitué jusqu'à $30 \%$ de la biomasse totale des ciliés lorsque les animaux ont reçu le régime "saccharose » et de 15 à $20 \%$ avec les régimes riches en inuline et lactose. P. multivesiculatum représente une part qui varie de 12 à $16 \%$ avec les régimes " inuline » et "saccharose », et devient négligeable avec le régime lactose $(1,1 \%)$.
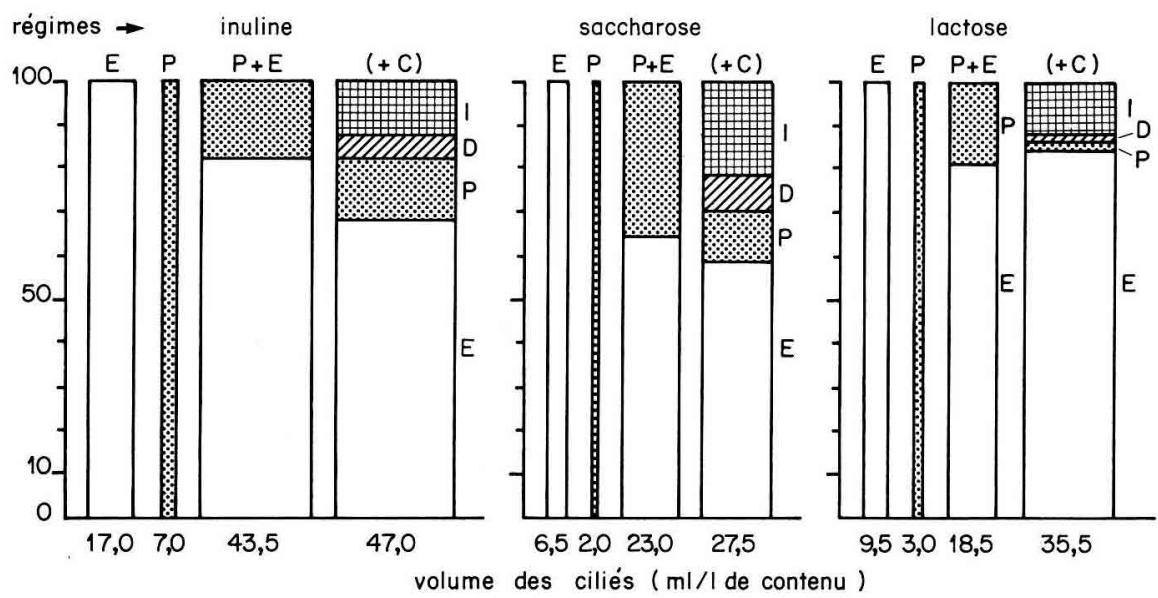

FIG. 1. - Influence du régime et du genre inoculé sur la biomasse des ciliés $(\mathrm{ml}$ de ciliés par litre de contenu\}.

Nature de la faune : $E=$ Entodinium ; $P=$ Polyplastron $; \mathrm{I}=$ Isotricha ; $\mathrm{D}=$ Dasytricha ; $(+\mathrm{C})=$ type conventionnel.

En ordonnée : proportion des différents genres présents. 


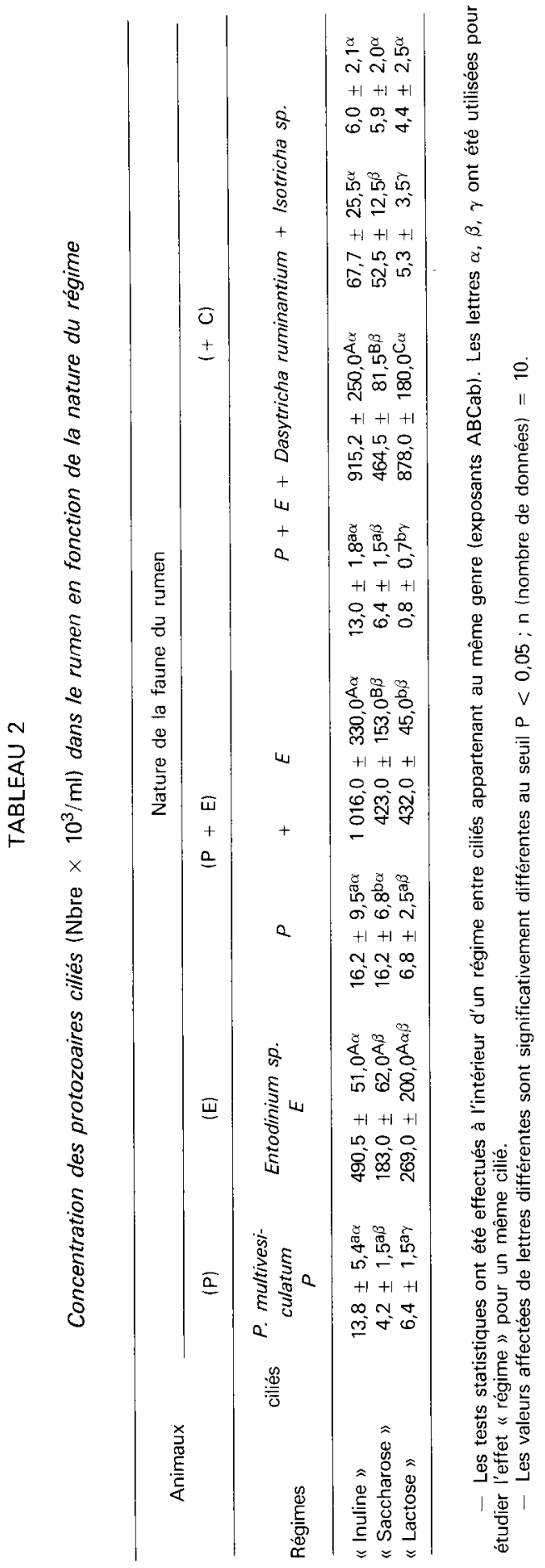


Dans le rumen des animaux inoculés avec les deux genres Polyplastron et Entodinium, la biomasse des ciliés a été importante avec le régime " inuline ", puisqu'elle a atteint des valeurs proches de celles mesurées chez les animaux conventionnels; elle a été environ deux fois plus faible chez les animaux qui recevaient du saccharose ou du lactose. La part représentée par Entodinium sp. a toujours été dominante dans la biomasse totale des ciliés ; elle a varié de $65 \%$ avec le régime " saccharose » à $80 \%$ avec les régimes "lactose " et " inuline ».

Chez les animaux mono-inoculés, l'effectif de chaque genre a été plus faible que celui mesuré chez les animaux bi-inoculés. La diminution a été particulièrement nette pour Entodinium sp. (jusqu'à $56 \%$ ) avec les trois régimes étudiés et pour $P$. multivesiculatum avec le régime "saccharose 》 (environ $74 \%$ ). En revanche, la biomasse de $P$. multivesiculatum a été peu différente chez les animaux mono- et bi-inoculés ( $P$ et $P+E$ ) qui ont reçu les régimes « inuline " et « lactose ».

\section{Utilisation digestive globale de la ration (tabl. 3 ).}

La présence du genre $P$. multivesiculatum dans le rumen a toujours amélioré la digestibilité apparente de la matière sèche et de la matière organique des rations étudiées. En revanche, l'effet positif d'Entodinium sp. n'a été significatif que dans le cas du régime "saccharose ». En combinant les deux genres de ciliés (Polyplastron + Entodinium sp.), on constate que l'action de Polyplastron est dominante, sauf dans le cas du régime "lactose ». Chez les animaux conventionnels, on retrouve généralement l'effet dû à Polyplastron.

L'utilisation digestive de la cellulose appréciée par la digestibilité de la lignocellulose a été, d'une manière générale, significativement améliorée par la présence de ciliés dans le rumen, et plus particulièrement par celle du genre Polyplastron. Un effet positif a également été observé chez les animaux monoinoculés avec le genre Entodinium $s p$. dans le cas des régimes "inuline » et

TABLEAU 3

Utilisation digestive apparente des principaux constituants des régimes et activité cellulolytique des bactéries en fonction de la nature de la faune du rumen

\begin{tabular}{lllllllc}
\hline \multicolumn{1}{c}{ Mesure (\%) } & Régime & $(-\mathrm{C})$ & $\mathrm{P}$ & $\mathrm{E}$ & $\mathrm{P}+\mathrm{E}$ & $(+\mathrm{C})$ & $\begin{array}{c}\text { Erreur } \\
\text { résiduelle }\end{array}$ \\
\hline CUD ap. NO & Inuline & $72,5^{\mathrm{a}}$ & $76,5^{\mathrm{b}}$ & $72,1^{\mathrm{a}}$ & $77,7^{\mathrm{b}}$ & $77,3^{\mathrm{b}}$ & 1,1 \\
& Saccharose & $74,5^{\mathrm{a}}$ & $76,3^{\mathrm{b}}$ & $78,4^{\mathrm{c}}$ & $77,7^{\mathrm{bc}}$ & $78,5^{\mathrm{c}}$ & 1,3 \\
& Lactose & $77,8^{\mathrm{a}}$ & $81,5^{\mathrm{b}}$ & $78,4^{\mathrm{a}}$ & $78,6^{\mathrm{a}}$ & $80,3^{\mathrm{b}}$ & 1,3 \\
\hline CUD ap. & Inuline & $66,4^{\mathrm{a}}$ & $68,2^{\mathrm{b}}$ & $68,3^{\mathrm{b}}$ & $67,7^{\mathrm{b}}$ & $68,7^{\mathrm{b}}$ & 1,3 \\
lignocellulose (ADF) & Saccharose & $62,5^{\mathrm{a}}$ & $67,1^{\mathrm{b}}$ & $66,4^{\mathrm{b}}$ & $69,4^{\mathrm{c}}$ & $69,2^{\mathrm{c}}$ & 2,0 \\
& Lactose & $55,4^{\mathrm{a}}$ & $58,6^{\mathrm{b}}$ & $54,7^{\mathrm{a}}$ & $57,9^{\mathrm{b}}$ & $58,9^{\mathrm{b}}$ & 1,8 \\
\hline Activité cellulolytique & Inuline & $29,9^{\mathrm{a}}$ & $25,6^{\mathrm{bc}}$ & $26,3^{\mathrm{b}}$ & $24,7^{\mathrm{c}}$ & $26,2^{\mathrm{b}}$ & 1,6 \\
des bactéries & Saccharose & $28,4^{\mathrm{a}}$ & $26,0^{\mathrm{b}}$ & $26,7^{\mathrm{b}}$ & $26,5^{\mathrm{b}}$ & $26,8^{\mathrm{ab}}$ & 1,9 \\
& Lactose & $28,1^{\mathrm{a}}$ & $26,8^{\mathrm{b}}$ & $26,2^{\mathrm{b}}$ & $27,2^{\mathrm{b}}$ & $23,2^{\mathrm{c}}$ & 1,2 \\
\hline
\end{tabular}

Les valeurs affectées de lettres différentes sur une même ligne sont significativement différentes au seuil de $P<0,05$. 
" saccharose ». Une action synergique, due à l'inoculation simultanée des deux genres chez les animaux bi-inoculés $P+E$ n'a été mise en évidence qu'avec le régime "saccharose ". La présence d'/sotrichidae en plus de Polyplastron et d'Entodinium dans le rumen des animaux conventionnels n'a pas modifié l'utilisation digestive de la lignocellulose observée chez les animaux $P+E$. L'activité cellulolytique des bactéries du rumen a généralement été réduite après l'inoculation de ciliés dans le rumen. Le genre de ciliés inoculé n'a eu que peu d'effet sur ce paramètre.

L'analyse factorielle (tabl. 4) confirme l'action positive de Polyplastron sur l'utilisation digestive de la matière organique et de la cellulose pour les trois régimes étudiés. L'effet global d'Entodinium sur ces deux paramètres n'est, par contre, pas significatif. Les deux genres diminuent l'activité cellulolytique des bactéries du rumen. La signification de l'effet est plus importante avec Polyplastron qu'avec Entodinium.

\section{Produits terminaux de la digestion dans le rumen (tabl. 4 et 5).}

Nous n'avons jamais observé d'évolution dans le volume du rumen entre les lots d'animaux étudiés. La valeur moyenne du contenu a été de 0,41 $\pm 0,04$; $0,39 \pm 0,02$ et $0,39 \pm 0,01$ litre par $\mathrm{kg}^{0,75}$ respectivement pour les animaux

\section{TABLEAU 4}

Signification des effets "protozoaires" et "régimes" ainsi que leurs interactions sur les principaux paramètres digestifs traités par analyse factorielle à 3 niveaux $(2 \times 2 \times 3)$

\begin{tabular}{|c|c|c|c|c|c|c|c|}
\hline & \multicolumn{7}{|c|}{ Signification des effets (Seuil de P) } \\
\hline & \multicolumn{4}{|c|}{ Effets simples } & \multicolumn{3}{|c|}{ Interactions } \\
\hline Paramètres & Polyplastron & Entodinium & Régime & $\begin{array}{l}\text { Répéti- } \\
\text { tions** }\end{array}$ & $\begin{array}{c}\text { Polyplas- } \\
\text { tron et } \\
\text { ento- } \\
\text { dinium } \\
\end{array}$ & $\begin{array}{l}\text { Polyplas- } \\
\text { tron-régime }\end{array}$ & $\begin{array}{l}\text { Entodi- } \\
\text { nium-régime }\end{array}$ \\
\hline CUD MO & $P<0,01$ & $\mathrm{P}<\mathrm{N} . \mathrm{S}$. & $P<$ N.S. & $P<$ N.S. & $P<N . S$. & $\mathrm{P}<\mathrm{N} . \mathrm{S}$. & $\mathrm{P}<\mathrm{N} . \mathrm{S}$. \\
\hline CUD ADF & 0,01 & N.S. & 0,01 & N.S. & N.S. & N.S. & N.S. \\
\hline $\begin{array}{l}\text { Activité cellulolytique } \\
\text { des bactéries du rumen }\end{array}$ & 0,01 & 0,05 & N.S. & N.S. & N.S. & N.S. & N.S. \\
\hline $\mathrm{pH}$ du jus de rumen & 0,01 & 0,01 & 0,01 & N.S. & 0,01 & N.S. & 0,05 \\
\hline $\begin{array}{l}\text { Concentration totale } \\
\text { A.G.V. jus de rumen }\end{array}$ & 0,01 & 0,05 & 0,01 & 0,05 & 0,01 & 0,05 & 0,01 \\
\hline $\mathrm{C}_{2}(\%$ molaire $)$ & 0,05 & 0,01 & 0,01 & N.S. & 0,05 & 0,01 & N.S. \\
\hline $\mathrm{C}_{3}(\%$ molaire $)$ & 0,05 & 0,01 & 0,01 & 0,05 & N.S. & 0,01 & N.S. \\
\hline $\mathrm{C}_{4}(\%$ molaire $)$ & 0,05 & N.S. & 0,01 & N.S. & 0,05 & 0,05 & N.S. \\
\hline $\mathrm{C}_{5}(\%$ molaire $)$ & N.S. & N.S. & N.S. & N.S. & 0,05 & N.S. & N.S. \\
\hline $\begin{array}{l}\text { Glucides alcoolo- } \\
\text { solubles (jus de rumen) }\end{array}$ & N.S. & N.S. & N.S. & N.S. & N.S. & N.S. & N.S. \\
\hline $\begin{array}{l}\text { Acide lactique } \\
\text { (jus de rumen) }\end{array}$ & N.S. & N.S. & N.S. & N.S. & N.S. & N.S. & N.S. \\
\hline $\mathrm{N}-\mathrm{NH}_{3}$ (jus de rumen) & 0,01 & N.S. & 0,01 & N.S. & N.S. ${ }^{*}$ & 0,01 & 0,05 \\
\hline Glycémie & N.S. & N.S. & 0,05 & N.S. & N.S. & N.S. & N.S. \\
\hline Hématocrites & N.S. & N.S. & N.S. & N.S. & N.S. & N.S. & N.S. \\
\hline
\end{tabular}

* Probabilité $P \simeq 0,05$.

* Correspond aux différences entre jours de prélèvement. 
TABLEAU 5

Produits de la fermentation dans le rumen en fonction de la nature de la faune

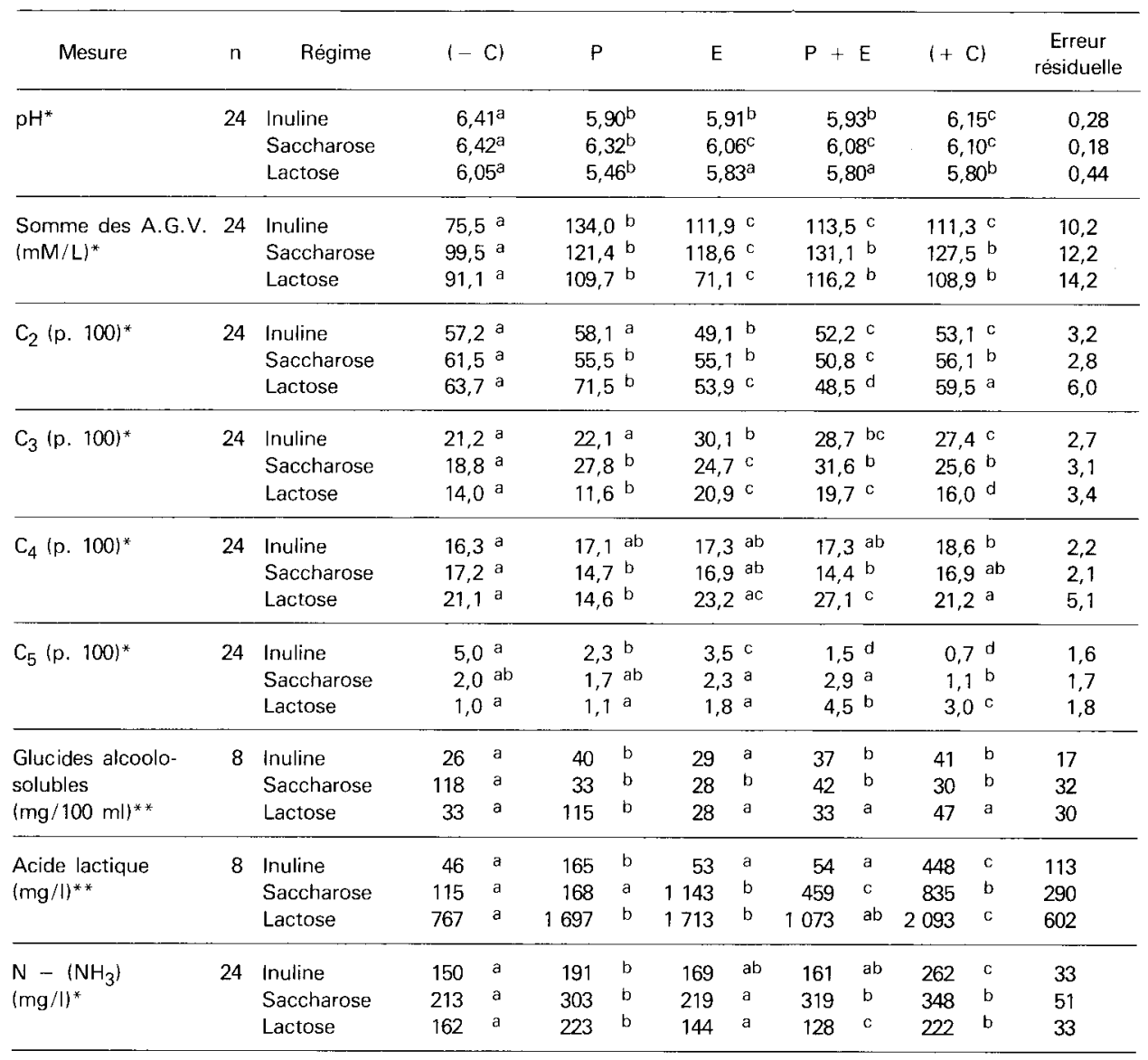

(Les valeurs affectées de lettres différentes sont significativement différentes au seuil $P<0,05$ ).

* Moyennes des prélèvements $t 1 / 2, \mathrm{t} 1 \mathrm{~h}, \mathrm{t} 1 \mathrm{~h} 30, \mathrm{t} 2 \mathrm{~h}, \mathrm{t} 3 \mathrm{~h}$ et $\mathrm{t} 5 \mathrm{~h}$ après les repas.

** Moyennes des prélèvements $t 1 \mathrm{~h} 30$ et $\mathrm{t} 3 \mathrm{~h}$ après les repas.

$\mathrm{n}=$ nombre de données.

ayant reçu les régimes " inuline », " saccharose » et "lactose ». En outre, les animaux avaient des poids vifs peu différents (de 37 à $44 \mathrm{~kg}$ ). Les variations de concentration des produits de la fermentation dans le rumen ne peuvent donc pas être dues à un effet de dilution, mais résultent bien d'une action métabolique globale des microorganismes du rumen, modifiée par la présence des ciliés.

Exception faite des animaux inoculés avec Entodinium sp. recevant le régime " lactose ", la concentration totale des acides gras volatils (AGV) dans le liquide du rumen a été significativement plus importante chez les animaux faunés que chez les animaux défaunés. C'est avec le genre Polyplastron présent dans le rumen des animaux, $P, P+E$ et $+C$ que l'effet a été le plus net (tabl. 4 et 5). Corrélativement, la valeur $\mathrm{du} \mathrm{pH}$ a toujours été significativement plus faible dans 
le rumen des animaux inoculés, plus particulièrement chez ceux dont la faune comprend l'espèce $P$. multivesiculatum.

La composition du mélange des AGV a été fréquemment modifiée par l'inoculation des ciliés. C'est la présence d'Entodinium chez les animaux $E, P+E$ et $+C$ qui a été à l'origine de l'action la plus nette sur les principaux AGV (tabl. 4 et 5) : elle a entraîné une diminution de 10 à $23 \%$ de la proportion molaire de l'acide acétique qui a été entièrement compensée par une augmentation de celle de l'acide propionique. L'action de Polyplastron sur ces paramètres a varié selon la nature du régime. Ce genre a entraîné des modifications identiques à celles d'Entodinium lorsque les animaux ont reçu le régime "saccharose "; l'évolution a été inverse avec le régime "lactose ", tandis qu'aucun effet significatif n'a pu être mis en évidence avec le régime « inuline ». L'augmentation de la proportion molaire de l'acide butyrique, habituellement observée après l'inoculation de ciliés dans le rumen, a été à la fois moins systématique et nettement plus faible pour les différents lots d'animaux étudiés; on a même observé une diminution du pourcentage d'acide butyrique dans le mélange des AGV chez tous les animaux faunés qui ont reçu le régime "saccharose ". L'analyse factorielle (tabl. 4) confirme l'effet nul d'Entodinium et négatif de Polyplastron sur la teneur des AGV en acide butyrique. Les ciliés Polyplastron et Entodinium n'ont eu que peu d'effet sur la proportion molaire de l'acide valérique et des acides ramifiés.

La concentration en acide lactique a toujours été plus importante dans le rumen des animaux faunés que chez les animaux défaunés. Toutefois, les écarts observés n'ont pas été significatifs avec les lots d'animaux $E$ et $P+E$ qui ont reçu le régime " inuline », ni avec les animaux $P$ et $P+E$ nourris respectivement avec les régimes " saccharose » et "lactose ". La concentration en glucides alcoolosolubles a légèrement augmenté chez les animaux faunés nourris avec le régime " inuline ", significativement diminué avec le régime " saccharose ", et peu évolué avec le régime "lactose». Toutefois, dans ce dernier cas, nous avons observé une augmentation importante de la concentration des glucides alcoolosolubles dans le rumen des animaux $P$. Le faible nombre de données $(N=8)$ et la dispersion des résultats n'ont pas permis de mettre en évidence un " effet ciliés » systématique sur ces paramètres.

La présence de $P$. multivesiculatum dans le rumen des animaux $P, P+E$ et $+C$ a toujours provoqué une augmentation fortement significative $(P<0,01)$ de la concentration en azote ammoniacal du jus de rumen (tabl. 4 et 5). En revanche, les animaux inoculés avec le seul genre Entodinium n'ont pas présenté de différence significative avec les animaux défaunés. L'analyse factorielle a permis de montrer l'absence d'effet d'Entodinium sur ce paramètre (tabl. 4). La présence d'une faune conventionnelle a presque toujours entraîné une augmentation de la concentration en azote ammoniacal par rapport à l'ensemble des autres lots d'animaux faunés.

Paramètres sanguins (tabl. 6).

A l'exception des lots $E$ et $+C$ recevant le régime "saccharose ", I'inoculation des ciliés dans des rumens défaunés n'a pas eu d'effet sur la glycémie. La valeur de l'hématocrite est restée constante tout au long de l'expérimentation 
Influence de la nature de la faune du rumen sur la glycémie et la valeur de l'hématocrite (n (nombre de données) $=4$ )

\begin{tabular}{llllllll}
\hline \multicolumn{1}{c}{ Mesures } & Régimes & (- C) & P & $E$ & $P+E$ & $(+C)$ & $\begin{array}{c}\text { Erreur } \\
\text { résiduelle }\end{array}$ \\
\hline Glycémie & Inuline & $67,1^{\mathrm{a}}$ & $69,1^{\mathrm{a}}$ & $64,1^{\mathrm{a}}$ & $69,9^{\mathrm{a}}$ & $68,5^{\mathrm{a}}$ & 7,1 \\
(mg/100 $\mathrm{ml})$ & Saccharose & $74,9^{\mathrm{a}}$ & $74,9^{\mathrm{a}}$ & $66,4^{\mathrm{b}}$ & $68,3^{\mathrm{ab}}$ & $60,8^{\mathrm{c}}$ & 7,5 \\
& Lactose & $58,8^{\mathrm{a}}$ & $63,0^{\mathrm{a}}$ & $59,7^{\mathrm{a}}$ & $62,0^{\mathrm{a}}$ & $63,8^{\mathrm{a}}$ & 5,3 \\
\hline Valeur de & Inuline & $35,8^{\mathrm{a}}$ & $33,6^{\mathrm{a}}$ & $33,0^{\mathrm{a}}$ & $34,3^{\mathrm{a}}$ & $39,3^{\mathrm{a}}$ & 4,1 \\
l'hématocrite & Saccharose & $35,5^{\mathrm{a}}$ & $36,0^{\mathrm{a}}$ & $33,3^{\mathrm{a}}$ & $36,1^{\mathrm{a}}$ & $33,7^{\mathrm{a}}$ & 3,0 \\
(p. 100) & Lactose & $32,9^{\mathrm{a}}$ & $32,8^{\mathrm{a}}$ & $35,8^{\mathrm{a}}$ & $33,5^{\mathrm{a}}$ & $36,2^{\mathrm{a}}$ & 2,0 \\
\hline
\end{tabular}

pour l'ensemble des animaux étudiés. L'analyse factorielle de ces deux paramètres a montré l'absence "d'effet ciliés "; seule la nature du régime a été à l'origine de modifications significatives de la glycémie.

\section{Discussion.}

Influence du régime sur le développement des ciliés.

Une bonne utilisation de l'énergie et de l'azote de la ration par les ciliés se traduit par un développement rapide de leur population, conduisant à un effectif final important. Parmi les trois régimes étudiés, dont la composition qualitative et quantitative en azote est voisine, c'est avec le régime " inuline " que l'effectif des populations de $P$. multivesiculatum et d'Entodinium $s p$. a été le plus dense chez les animaux mono-inoculés. Abou Akkada et al. (1963) avaient montré in vitro que $P$. multivesiculatum possède une invertase capable d'hydrolyser le saccharose, le raffinose et l'inuline. La confirmation in vivo de ce résultat s'explique par le développement important de ce cilié avec le régime « inuline ». Sa plus faible concentration avec le régime "saccharose " reste toutefois difficile à expliquer. En outre, P. multivesiculatum est capable d'utiliser les substances pectiques (Abou Akkada et al., 1963) présentes en quantité appréciable dans les betteraves (Jarrige et Fauconneau, 1973) et dans les topinambours oú elles représentent $2,1 \%$ de la matière sèche (Jouany, données non publiées). C'est également, la seule espèce de ciliés qui possède une lactase active, capable d'hydrolyser le lactose (Abou Akkada et al., 1963). L'équipement enzymatique complet de ce cilié, qui lui permet également de digérer la cellulose (Jouany et Senaud, 1982), lui confère un intérêt tout particulier dans la digestion des glucides présents dans l'alimentation du Ruminant.

Les travaux réalisés in vitro par Abou Akkada et Howard (1960) ainsi que Williams et al. (1960) ont montré qu'Entodinium sp. n'utilise pas les glucides solubles. Cependant, en 1962, Coleman a indiqué que la majeure partie des espèces appartenant à ce genre peut métaboliser les sucres, à condition qu'elles soient à jeun ; le même auteur a ensuite précisé (Coleman, 1969) qu'Entodinium caudatum, in vitro, mis en présence de ${ }^{14} \mathrm{C}$-saccharose, est capable d'incorporer $\mathrm{du}{ }^{14} \mathrm{C}$ dans ses réserves glucidiques. L'effectif d'Entodinium $s p$. a toujours été 
plus faible avec le régime "saccharose ", aussi bien chez les animaux mono, biinoculés, que chez les animaux conventionnels. Ce résultat peut indiquer que le genre Entodinium $s p$. ne métabolise pas rapidement ce sucre pour assurer son propre développement. La présence d'un effectif moyen d'Entodinium $s p$. chez les animaux mono-inoculés nourris avec le régime " inuline " ne nous permet pas d'affirmer qu'Entodinium sp. peut fermenter ce glucide ; il pourrait également métaboliser le fructose et surtout le glucose résultant de l'hydrolyse du saccharose par les bactéries. Les études qui avaient été entreprises sur l'équipement enzymatique d'Entodinium sp. n'avaient pas permis de mettre en évidence l'existence d'une lactase (Howard, 1957 ; Abou Akkada et Howard, 1960 ; Kubo et Kandatsu, 1968). II est donc probable que ce cilié n'utilise pas directement le lactose, mais plutôt les hexoses (glucose et galactose) résultant de son hydrolyse par les bactéries ou les autres ciliés. Cette hypothèse est confirmée par l'augmentation de sa population lorsqu'il est présent simultanément avec d'autres espèces qui peuvent hydrolyser le lactose (cas des animaux $P+E$ et $+C$ ).

Le développement des /sotrichidae (/sotricha $s p$. et $D$. ruminantium) dans le rumen est également lié étroitement à l'utilisation des 3 substrats glucidiques étudiés ou aux oses résultant de leur hydrolyse, qui, de par leur solubilité, peuvent être métabolisés par cette famille de ciliés (cf. Hungate, 1966). L'importance des populations d'/sotricha $s p$. et de Dasytricha ruminantium avec les régimes « inuline " et "saccharose " est probablement due à une utilisation directe de ces deux glucides, car de nombreuses études réalisées in vitro ont montré que ces ciliés possèdent une invertase active qui leur permet de métaboliser ces glucides aussi rapidement que des oses libres (Heald et Oxford, 1953 ; Howard, 1959b ; Punj et al., 1970). Selon Mould et Thomas (1958), les deux genres /sotricha et Dasytricha auraient des activités invertasiques identiques. En revanche, Howard (1959a et b) a montré qu'/sotricha était plus actif que Dasytricha dans la production de cet enzyme. Nos études réalisées in vivo indiquent (tabl. 2), à partir des effectifs respectifs, que l'activité de Dasytricha serait supérieure à celle d'/sotricha. Ces mêmes données, exprimées non plus en concentration de cellules par unité de volume mais en biomasse (fig. 1), mettent en valeur /sotricha dont le volume est environ 30 fois supérieur à celui de Dasytricha. D'après Howard (1959a et b), Prins et Van Hoven (1977) ; Van Hoven et Prins (1977), aucun des deux genres n'est capable d'utiliser directement la molécule de lactose, mais seulement les oses libérés par son hydrolyse. Les effectifs voisins des deux genres avec le régime "lactose " indiquent qu'ils utilisent probablement les mêmes sources de carbone provenant de la dégradation du lactose par les bactéries ou par les autres ciliés.

\section{Action des ciliés sur les fermentations dans le rumen.}

L'utilisation digestive apparente de la matière organique des régimes étudiés a été supérieure en présence des ciliés. L'effet a été particulièrement net avec le genre Polyplastron. Nous confirmons ici des résultats obtenus antérieurement (Jouany et al., 1981a ; Jouany et Senaud, 1982).

L'activité fermentaire dans le rumen, appréciée à la fois par la valeur du $\mathrm{pH}$ et par la concentration en AGV du contenu, a généralement été plus importante 
chez les animaux faunés que chez les défaunés. Ceci traduit le rôle positif que jouent les ciliés dans le métabolisme de ces différents glucides et confirme les résultats des nombreux travaux effectués sur la comparaison de la digestion dans le rumen entre animaux faunés et animaux défaunés (Abou Akkada et El Shazly, 1964 ; Christiansen et al., 1965 ; Klopfenstein et al., 1966 ; Luther et al., 1966 ; Borhami et al., 1967 ; Kurihara et al., 1968 ; Youssef et Allen, 1968 ; Eadie et Gill, 1971 ; Itabashi et Kandatsu, 1975). L'augmentation de I'intensité des fermentations en présence des ciliés correspond vraisemblablement à une plus grande quantité d'AGV produite dans le rumen. II n'est toutefois pas possible de déterminer si cela correspond à une production propre d'AGV par les protozoaires ciliés comme l'ont montré in vitro Hungate et al. (1952), Gutierrez (1955, 1958) et Hungate (1960), ou bien à une stimulation du métabolisme des bactéries due à la prédation des protozoaires sur les bactéries dans le rumen des animaux faunés (Kurihara et al., 1968). Il est probable que les deux phénomènes interviennent simultanément. Nous avons également constaté que l'action spécifique des genres Polyplastron et Entodinium sur la concentration des AGV n'est pas très différente de l'action d'une faune conventionnelle. Il est difficile d'expliquer le fort ralentissement de l'activité fermentaire observé avec le régime "lactose » chez les animaux ayant le seul genre Entodinium. Une partie importante de ce substrat énergétique a pû être métabolisée par les bactéries pour former du lactate dont la présence a été difficile à mettre en évidence par suite de l'espacement des prélèvements après le repas.

L'influence des ciliés sur la composition du mélange des AGV a été moins systématique. L'augmentation de la proportion molaire de l'acide butyrique généralement observée après l'inoculation de protozoaires ciliés dans le rumen (cf. revue dans Jouany et al., 1981a) a été faible, voire nulle, avec les trois régimes étudiés. L'évolution des autres principaux AGV difficile à caractériser à partir des données bibliographiques, a été par contre plus régulière au cours de cette étude : la proportion molaire d'acide propionique a été généralement accrue chez les animaux faunés, aux dépens essentiellement de celle de l'acide acétique.

L'analyse statistique factorielle à trois niveaux (tabl. 4) a permis de mettre en évidence l'effet spécifique de certains ciliés sur la composition du mélange des AGV. Nous avons pu montrer que $P$. multivesiculatum a eu une action spécifique sur ce paramètre et que celle-ci a varié en fonction de la nature du régime. En revanche, l'effet d'Entodinium sur les acides acétique et propionique a été indépendant de la source de glucide étudié. Enfin, nous avons constaté, par cette méthode d'analyse, qu'il n'y avait pas de différence significative entre les jours de prélèvement ( $($ répétitions », dans tableau 4).

Nous avons souvent noté la présence d'acide lactique en plus grande quantité dans le rumen des animaux faunés sans qu'il soit possible de relier ce paramètre à la nature de la faune (tabl. 4). Les travaux de Heald et Oxford (1953), de Gutierrez (1955) et de Howard (1959a) réalisés in vitro ont montré que les ciliés produisent de I'acide lactique. Il pourrait donc s'agir là d'un effet " ciliés " direct sur la production de ce métabolite qui est un précurseur important de l'acide propionique (Ekern et Reid, 1963), lequel est également présent en plus grande quantité chez les animaux faunés. 
A l'exception du seul lot d'animaux $E$, la concentration en azote ammoniacal a toujours été plus importante après l'inoculation des protozoaires ciliés dans un rumen défauné. Plusieurs auteurs ont précisé in vitro que les ciliés dégradent les protéines (Warner, 1955, 1956 ; Hungate, 1966) pour former des acides aminés et des peptides à chaînes courtes (Abou Akkada et Howard, 1962). Au cours de la protéolyse, la production d'ammoniaque serait limitée puisque selon Abou Akkada (1965), seulement $10 \%$ de l'azote dégradé apparaîtrait sous forme ammoniacale. En outre, l'ammoniaque ne serait pas formé par désamination des acides aminés, mais proviendrait essentiellement de l'hydrolyse des groupements amides par les ciliés, ou bien du métabolisme endogène des ciliés lorsque ces derniers sont à jeûn (Abou Akkada et Howard, 1962). Comme Males et Purser (1970), on peut penser que l'augmentation de la concentration en ammoniaque du jus de rumen provient de son utilisation plus faible par les bactéries qui sont moins nombreuses dans le rumen des animaux faunés (Eadie et Hobson, 1962 ; Kurihara et al., 1968 ; Eadie et Gill, 1971 ; Williams et Dinusson, 1972 ; Monghal, 1975 ; Collombier, 1981). Cette hypothèse est apparemment en contradiction avec les résultats de Itabashi et Kandatsu (1975) qui ont montré que l'utilisation du ${ }^{15} \mathrm{~N}-\mathrm{NH}_{3}$ par les bactéries était identique en présence ou en l'absence de protozoaires.

\section{Action des ciliés sur la digestion de la cellulose.}

Nous avons montré, au cours de ce travail, que la présence de ciliés (surtout, $P$. multivesiculatum) dans le rumen, améliore toujours la digestibilité de la lignocellulose.

Si la digestibilité globale de la cellulose ne nous renseigne pas sur le site de sa digestion, on peut toutefois penser, comme Demeyer (1981), qu'une réduction de la digestibilité de la cellulose dans le rumen chez les animaux défaunés est compensée par une proportion plus importante de fibres digérées dans le gros intestin. Ce déplacement de la digestion réduit l'écart observé entre les animaux défaunés et faunés. On peut donc émettre l'hypothèse que l'amélioration de la digestibilité mesurée chez les animaux faunés aurait été encore plus importante si la mesure avait été effectuée à la sortie des estomacs.

Les bactéries sont en partie responsables de cette amélioration lorsque les animaux reçoivent une ration riche en cellulose (Jouany et Senaud, 1982) puisque leur activité cellulolytique est accrue à la suite de l'introduction de ciliés dans le rumen. Par contre, lorsque les régimes ont une teneur faible en cellulose, les ciliés semblent être seuls responsables de ce phénomène puisque l'activité cellulolytique des bactéries est plus faible chez les animaux faunés. Il est bien admis (cf. Hungate, 1966) que la composition qualitative de la flore du rumen évolue avec la nature de l'énergie de la ration et que la flore cellulolytique est systématiquement plus abondante avec les régimes à base de fourrages grossiers qu'avec les régimes " concentrés ». La présence de protozoaires aura donc proportionnellement plus d'effet sur la diminution de la flore cellulolytique dans le cas des régimes riches en énergie facilement fermentescible. La faune, dont la composition qualitative varie peu d'un régime à l'autre (fig. 1), peut alors jouer un rôle important dans la dégradation de la cellulose lorsque celle-ci est ingérée en quan- 
tité limitée. Dans ces conditions, la faible activité cellulolytique d'Entodinium $s p$. mise en évidence par Bonhomme-Florentin (1975) devient significative. Enfin, nous avons confirmé dans ce travail le rôle cellulolytique de $P$. multivesiculatum que nous avons déjà observé antérieurement (Jouany et Senaud, 1979 ; Jouany et al., 1981a, b ; Jouany et Senaud, 1982).

\section{Conclusion.}

Les ciliés modifient le biotope qu'est le rumen ainsi que l'écosystème microbien constitué de l'ensemble «protozoaires et bactéries". Les répercussions observées sur la digestion de différentes rations sont généralement importantes. II n'est pas toujours possible, dans l'état actuel de nos connaissances, de préciser s'il s'agit d'un effet direct des ciliés ou du résultat de modifications profondes sur la flore du rumen. Dans le cas où les deux actions seraient conjuguées, ce qui est probable, nous ne sommes actuellement pas capables de déterminer la part qui revient à chacune d'elles. Enfin, l'isolement in vivo des deux principaux genres rencontrés dans notre région (Po/yplastron et Entodinium) a permis de mettre en évidence des différences importantes dans leur métabolisme respectif à l'égard de substrats glucidiques variés (amidon, saccharose, inuline, lactose et cellulose). Ainsi, nous avons pu constater que $P$. multivesiculatum possède un équipement enzymatique très diversifié qui lui permet d'utiliser pratiquement toutes les sources glucidiques présentes dans la ration du Ruminant. Entodinium $s p$. , dont la production d'enzymes est plus limitée, peut se développer dans le rumen en utilisant les produits de dégradation résultant de l'activité fermentaire des autres ciliés ou des bactéries. Ce résultat met en évidence l'importance des inter-relations entre ciliés d'une part, et entre ciliés et bactéries d'autre part, lesquelles ont déjà été discutées par Eadie $(1962,1967)$ et complétées par nos propres observations (Jouany, 1978 ; Senaud et al., 1980 ; Grolière et al., 1980).

Reçu en septembre 1982. Accepté en janvier 1983.

Remerciements. - Nous tenons à remercier Mmes Marie-Paule Girard et Bernadette Lassalas ainsi que Melles Marie-Thérèse Beaufort et Paulette Journaix pour leur aide au laboratoire, M. J. Lefaivre pour la réalisation des interventions chirurgicales, enfin MM. L. L'Hotelier, C. Marpillat et A. Vizet pour le soin qu'ils ont apporté à l'entretien des animaux au cours de la réalisation de ce travail.

\section{References}

ABOU AKKADA A. R., 1965. The metabolism of ciliate protozoa in relation to rumen function, 335-345. In DOUGHERTY R. W., Physiology of digestion in the ruminant, Butterworths, London.

ABOU AKKADA A. R., EADIE J. M., HOWARD B. H., 1963. The biochemistry of rumen protozoa : 7. The carbohydrases of Polyplastron multivesiculatum. Biochem. J., 89, 268272. 
ABOU AKKADA A. R., EL SHAZLY K., 1964. Effect of absence of ciliate protozoa from the rumen on microbial activity and growth of lambs. Appl. microbiol., 12, 384-390.

ABOU AKKADA A. R., HOWARD B. H., 1960. The biochemistry of rumen protozoa: the carbohydrate metabolism of Entodinium. Biochem. J., 76, 445-451.

ABOU AKKADA A. R., HOWARD B. H., 1962. The biochemistry of rumen protozoa : the nitrogen metabolism of Entodinium. Biochem. J., 82, 313-320.

BONHOMME-FLORENTIN A., 1975. Activité cellulolytique des ciliés " Entodiniomorphes ». J. Protozool., 22, 447-451.

BORHAMI B. E. A., EL SHAZLY K., ABOU AKKADA A. R., AMHED I. A., 1967. Effect of early establishment of ciliate protozoa in the rumen on microbial activity and growth of early weaned buffalo calves. J. Dairy Sci., 50, 1654-1660.

CHRISTIANSEN W. C., KAWASHIMA R., BURROUGHS W., 1965. Influence of protozoa upon rumen acid production and liveweight gains in lambs. J. anim. Sci., 24, 730-734.

COLEMAN G. S., 1962. The preparation and survival of bacteria-free suspensions of Entodinium caudatum. J. gen. Microbiol., 28, 271-281.

COLEMAN G. S., 1969. The metabolism of starch, maltose, glucose and some other sugars by the rumen ciliate Entodinium caudatum. J. gen. Microbiol., 57, 303-332.

COLLOMBIER J., 1981. Contribution à l'étude du rôle des protozoaires ciliés du rumen dans l'apport d'azote microbien entrant dans le duodénum du Ruminant. Th. Doct.-Ing., Univ. Clermont $I I, n^{\circ}$ d'ordre 105, $85 \mathrm{pp}$.

DEMEYER D. I., 1981. Rumen microbes and digestion of plant cell walls. Agr. Environ., 6, 295-337.

EADIE J. M., 1962. Inter-relationship between certain rumen ciliate protozoa. J. gen. Microbiol., 29, 579-588.

EADIE J. M., 1967. Studies on the ecology of certain rumen ciliate protozoa. J. gen. Microbiol., 49, 175-194.

EADIE J. M., GILL J. C., 1971. The effect of the absence of rumen ciliate protozoa on growing lambs fed on roughage-concentrate diet. Br. J. Nutr., 26, 155-167.

EADIE J. M., HOBSON P. N., 1962. Effect of presence or absence of ciliates on the total rumen bacterial count in lambs. Nature, 193, 503-505.

EKERN A., REID J. T., 1963. Efficiency of energy utilization by young cattle ingesting diets of hay, silage and hay supplemented with lactic acid. J. dairy Sci., 46, 522-529.

GROLIERE C. A., SENAUD J., JOUANY J. P., GRAIN J., DE PUYTORAC P., 1980. Implantation et développement des populations de protozoaires ciliés (Polyplatron multivesiculatum, Entodinium sp., Isotricha prostoma/ dans le rumen de moutons recevant différents régimes alimentaires. II. Régimes à base de foin, enrichis en céréales. Protistologica, 16, 385-394.

GUTIERREZ J., 1955. Experiments on the culture and physiology of " holotrichs " from the bovine rumen. Biochem. J., 60, 516-522.

GUTIERREZ J., 1958 . Observations on bacterial feeding by the rumen ciliate /sotricha prostoma. J. Protozool., 5, 122-126.

HEALD P. J., OXFORD A. E., 1953. Fermentation of soluble sugars by anaerobic " Holotrich" ciliate protozoa of the genera /sotricha and Dasytricha. Biochem. J., 53, 506-512.

HOWARD B. H., 1957. Biochemical studies of individual genera of rumen " Holotrich " protozoa. Biochem. J., 67, $18 \mathrm{P}$ (Abstr.).

HOWARD B. H., 1959a. The biochemistry of rumen protozoa. 1. Carbohydrate fermentation by Dasytricha and /sotricha. Biochem. J., 71, 671-675.

HOWARD B. H., 1959b. The biochemistry of rumen protozoa. 2. Some carbohydrases in cell-free extracts of Dasytricha and Isotricha. Biochem. J., 71, 675-680.

HOWARD B. H., 1963. Hydrolysis of naturally occuring galactosides by some rumen protozoa. Biochem. J., 89, 90 P-91 P (Abstr.).

HUNGATE R. E., 1960. Symposium : selected topics in microbial ecology. I. Microbial ecology of the rumen. Bact. Rev., 24, 353-357.

HUNGATE R. E., 1966. The rumen and its microbes. Acad. Press, New York, San Francisco, London.

HUNGATE R. E., DOUGHERTY W., BRYANT M. P., CELLO R. M., 1952. Microbiological and physiological changes associated with accute indigestion in sheep. Corne/l Vet., 42, 423-427. 
ITABASHI H., KANDATSU M., 1975. Influence of rumen ciliate protozoa on the concentration of ammonia and volatile fatty acids in connection with the utilization of ammonia in the rumen. Jap. J. zootechn. Sci, 46, 409-416.

JARRIGE R., 1961. Analyse des constituants glucidiques des plantes fourragères. I. Fractionnement des constituants de la membrane par les hydrolyses acides. Ann. Biol. anim. Bioch. Biophys., 1, 163-212.

JARRIGE R., FAUCONNEAU G., 1973. Les constituants glucidiques et azotés des racines de betteraves de différents types. Ann. Biol. anim. Bioch. Biophys., 13, 141-146.

JOUANY J. P., 1972. Chromatographie en phase gazeuse des mono- di- et triholosides dans les milieux complexes. Ann. Biol. anim. Bioch. Biophys., 12, 493-504.

JOUANY J. P., 1978. Contribution à l'étude des protozoaires ciliés du rumen : leur dynamique, leur rôle dans la digestion et leur intérêt pour le Ruminant. Th. Doct. Etat, $\mathrm{n}^{\circ} \mathrm{d}^{\prime}$ ordre 256. Univ. Clermont II, 195 pp.

JOUANY J. P., SENAUD J., 1979. Role of rumen protozoa in the digestion of food cellulosic materials. Arir. Rech. vét., 10, 261-263.

JOUANY J. P., SENAUD J., 1982. Influence des ciliés du rumen sur la digestion de différents glucides chez $I \in$ Mouton. I. Utilisation des glucides pariétaux (cellulose et hémicelluloses) et de l'amidon. Reprod. Nutr. Dévelop., 22, 735-752.

JOUANY J. P., ZAINAB B., SENAUD J., GROLIERE C. A., GRAIN J., THIVEND P., 1981a. Role of the rumen ciliate protozoa Polyplastron multivesiculatum, Entodinium $s p$. and /sotricha prostoma in the digestion of a mixed diet in sheep. Reprod. Nutr. Develop., 21, 871-884.

JOUANY J. P., SENAUD J., GROLIERE C. A., THIVEND P., GRAIN J., 1981b. Influence du traiternent par la soude et de l'inoculation du cilié Polyplastron multivesiculatum sur la digestion d'un régime riche en glucides pariétaux. Reprod. Nutr. Dévelop., 21, 866 (abstr.).

KLOPFENSTE:H, T. J., PURSER D. B., TYZNIK W. J., 1966. Effects of defaunation on feed digestibility, rumen metabolism and blood metabolites. J. anim. Sci., 25, 765-773.

KUBO T., KANDATSU M., 1968. Carbohydrate metabolism of rumen ciliate protozoa. I. Utilization of soluble sugars by mixed rumen protozoa. N.A.R., 39, 1241 (Abstr.).

KURIHARA Y., EADIE J. M., HOBSON P. N., MANN S. O., 1968. Relationship between bacteria and ciliate protozoa in the sheep rumen. J. gen. Microbiol., 51, 267-288.

LUTHER R., TRENKLE A., BURROUGHS W., 1966. Influence of rumen protozoa on volatile acid production and ration digestibility in lambs. J. anim. Sci, 25, 1116-1122.

MALES J. R., PURSER D. B., 1970. Relationship between rumen ammonia levels and the microbial population and volatile fatty acid proportions in faunated and defaunated sheep. Appl. Microbiol., 19, 485-490.

MONGHAL M., 1975. Contribution à l'étude des interrelations entre ciliés et bactéries dans le rumen de mouton. Th. 3e cycle. Univ. Clermont II. $\mathrm{N}^{\circ}$ d'ordre $454.58 \mathrm{pp}$.

MOULD D. L., THOMAS G. J., 1958. The enzymatic degradation of starch by "holotrichs » from sheep rumen. Biochem. J., 69, 327-337.

PRINS R. A., VAN HOVEN W., 1977. Carbohydrate fermentation by the rumen ciliate Isotricha prostoma. Protistologica, 13, 549-556.

PUNJ M., KOCHAR A. S., BHATIA I., 1970. Degradation and metabolism of inuline by rumen microorganisms. Ind. J. Nutr. Dietetics, 7, 247-251.

SENAUD J., GROLIERE C. A., ZAINAB B., GRAIN J., JOUANY J. P., 1980 . Implantation et développement des populations de protozoaires ciliès (Polyplastron multivesiculatum, Entodinium sp., Isotricha prostoma) dans le rumen de moutons recevant différents régimes alimentaires. I. Régime luzerne-orge (40/50). Prostistologica, 16, 325-337.

SNEDECOR G. W., COCHRAN W. G., 1971. Méthodes statistiques, 6 éd. A.C.T.A. ed. Paris.

THIVEND P., 1978. Le lactosérum dans I'alimentation du Ruminant. Bull. techn. CRZV Theix, 31, 11-17.

VAN HOVEN W., PRINS R. A., 1977. Carbohydrate fermentation by the rumen ciliate Dasytricha ruminantium. Protistologica, 13, 595-606.

WARNER A. C. I., 1955. Some aspects of the nitrogen metabolism of the microorganisms of the rumen with special reference to proteolysis. Ph. D. Thes., University of Aberdeen, Scotland. 
WARNER A. C. I., 1956. Proteolysis by rumen microorganisms. J. gen. Microbiol., 28, 129-146. WILLIAMS P. P., GUTIERREZ J., DOETSCH R. N., 1960, cité par HUNGATE (1966).

WILLIAMS P. P., DINUSSON W. E., 1972. Amino acid and fatty acid composition of bovine ruminal bacteria and protozoa. J. anim. Sci., 36, 151-155.

YOUSSEF F. G., ALLEN D. M., 1968. Role played by ciliate protozoa in rumen function. Nature, 217, 777-778. 